Research Paper

\title{
Elevated miR-34c-5p Mediates Dermal Fibroblast Senescence by Ultraviolet Irradiation
}

Bing-rong ZHOU*, Xian-fei GUO*, Jia-an ZHANG, Yang XU, Wei LI, Di WU, Zhi-qiang YIN, Felicia Permatasari and Dan LUO ${ }^{\bowtie}$

Department of Dermatology, the First Affiliated Hospital of Nanjing Medical University, Guangzhou road 300\#, Nanjing, Jiangsu province, China PR.

* contribute equally to the paper.

$\triangle$ Corresponding author: E-Mail: daniluo2013@njmu.edu.cn (D.L.); Tel.: +86-25-8679-6545; Fax: +86-25-8371-8836.

() Ivyspring International Publisher. This is an open-access article distributed under the terms of the Creative Commons License (http://creativecommons.org/ licenses/by-nc-nd/3.0/). Reproduction is permitted for personal, noncommercial use, provided that the article is in whole, unmodified, and properly cited.

Received: 2012.10.06; Accepted: 2013.07.23; Published: 2013.08.09

\begin{abstract}
Previous studies showed that several miRNAs can regulate pathways involved in UVB-induced premature senescence and response to ultraviolet irradiation. It has also been reported that miR-34c-5p may be involved in senescence-related mechanisms. We propose that miR-34c-5p may play a crucial role in senescence of normal human primary dermal fibroblasts. Here, we explored the roles of miR-34c-5p in UVB-induced premature senescence on dermal fibroblasts. MiR-34c-5p expression was increased in dermal fibroblasts after repeated subcytotoxic UVB treatments. Underexpression of miR-34c-5p in dermal fibroblasts led to a marked delay of many senescent phenotypes induced by repeated UVB treatments. Furthermore, underexpression of miR-34c-5p in dermal fibroblasts can antagonize the alteration of GI-arrested fibroblasts. Moreover, E2F3, which can inactivate $\mathrm{p} 53$ pathway and play a role in cell cycle progression, is a down-stream target of miR-34c-5p. Forced down-expression of miR-34c-5p decreased the expression of UVB-SIPS induced P2I and P53 at both mRNA and protein levels. Our data demonstrated that down-regulation of miR-34c-5p can protect human primary dermal fibroblasts from UVB-induced premature senescence via regulations of some senescence-related molecules.
\end{abstract}

Key words: miR-34c-5p; UVB; premature senescence; human skin fibroblasts.

\section{Introduction}

Premature senescence of human dermal fibroblasts can be induced by exposures to a variety of oxidative stress and DNA damaging agents. Debacq-Chainiaux F. et al. developed a robust model of UVB-induced premature senescence of human dermal fibroblasts (UVB-SIPS)(1). This model represents an alternative in vitro model in photoaging research for investigating photoaging-related mechanisms(2).

MicroRNAs (miRNAs) have emerged recently as a new class of small evolutionarily conserved non-coding RNAs that negatively regulate gene expression. Several miRNAs have been shown to be involved in the regulation of pathways involved in
SIPS and response to ultraviolet irradiation $(3,4)$. MiR-34c-5p has been reported to mediate growth arrest and upregulation of the percentage of aging cells in a variety of cell types(5). Kyle Lafferty-Whyte et al. also reviewed that miR-34c had the potential to regulate all 4 kinds of senescence induction types (replicative senescence, oxidative stress, oncogene expression, and DNA damage signaling). Their study highlights future potential of miR-34c as novel drug targets for senescence induction(6).

Two mature miRNA species are derived from the miR-34c precursor, namely miR-34c-5p and miR-34c-3p. However, they have different seed se- 
quences that regulate different targets. In the present study, we found that miR-34c-5p was overexpressed in UVB-SIPS fibroblasts by means of miRNA microarray, and further confirmed by real-time quantitative PCR (qRT-PCR). In consideration of the functions of miR-34c mentioned above, we speculate that miR-34c-5p might play very important roles in cellular senescence, which needs further study.

\section{Experimental Section}

\section{Cultivation of primary human dermal fibro- blasts and UV light source}

Primary human dermal fibroblasts were obtained from four Chinese donors aged 8-12 years by means of a foreskin circumcision, and cells were cultured by the same method previously used (7). For the experiments, human dermal fibroblasts used were between passage 2-4. The source of UVB was BLE-1T158 (Spectronics Corp., Westbury, NY, USA). A Kodacel filter (TA401/407, Kodak, Rochester, USA) was used to block wavelengths of less than $290 \mathrm{~nm}$ (ultraviolet C). The UVB dosage was quantified using a Waldmann UV meter (model no. 585100: Waldmann Co., VS-Schwenningen, Germany).

\section{Induction of UVB-SIPS and SA- $\beta$-gal activity detection}

$25 \mathrm{~mJ} / \mathrm{cm}^{2} \mathrm{UVB}$ was performed twice a day for 5 days. Control cells were kept in the same culture conditions without UVB exposure. At 48 hours after the last stress, the SA- $\beta$-gal activity was assessed with SA- $\beta$-gal Staining kit (Biotime, Haimen, China) by using the method originally described by $\mathrm{Oh}$ et al(8).

\section{RNA isolation and miRNA microarray}

Total RNA isolation and miRNA enrichment were performed with a mirVana miRNA Isolation Kit (Ambion, Austin, TX, USA) according to the manufacturer's instructions. RNA concentration was quantified with a NanoDrop spectrophotometer (Thermo Fisher, Waltham, MA, USA). RNA integrity was evaluated using an Agilent 2100 Bioanalyzer (Agilent Technologies, Santa Clara, CA, USA). RNA labeling and hybridization on the Agilent miRNA microarray chips were performed with an miRNA Labeling Reagent and Hybridization Kit (Agilent Technologies, Santa Clara, CA, USA) at $37^{\circ} \mathrm{C}$ for $30 \mathrm{~min}$.

Each total RNA sample (100 ng) was treated with calf intestine alkaline phosphatase (TaKaRa, Dalian, China), denatured using 100\% dimethyl sulfoxide (Sigma, Taufkirchen, Germany) at $100{ }^{\circ} \mathrm{C}$ for $8 \mathrm{~min}$ in a thermal cycler, and then transferred to an ice-water bath to prevent RNA from re-annealing. The RNA samples were then labeled with $\mathrm{pCp}-\mathrm{Cy} 3$ using $\mathrm{T} 4$
RNA ligase (Ambion, Austin, TX, USA) via incubation at $16^{\circ} \mathrm{C}$ for $2 \mathrm{~h}$. The labeled samples were hybridized to Agilent human miRNA microarrays, which contained probes for 1223 miRNAs cataloged in the Sanger Cambridge database v10.1 (http://microrna. sanger.ac.uk). Hybridizations were performed in SureHyb chambers (Agilent Technologies, Santa Clara, CA, USA) for $24 \mathrm{~h}$ at $55^{\circ} \mathrm{C}$. The microarrays were then washed using Agilent-prepared buffers.

The microarray images were scanned with an Agilent microarray scanner, gridded, and analyzed using Agilent Feature Extraction Software version 9.5.1 (Santa, Clara, CA, USA). Normalization was performed using the per-chip median normalization method and the median array (9).

\section{Reverse-transcription real-time PCR analysis}

Expression of individual miRNAs was detected by qRT-PCR using miRNA sequence-specific primers (Applied Biosystems, Foster City, CA). Briefly, 10 ng of total RNA was reverse-transcribed using a High-Capacity cDNA Archive kit (Applied Biosystems) followed by amplification in an ABI 7500 Real-Time PCR System (Applied Biosystems, Santa Clara, CA, USA). All RT-PCRs were performed in triplicate. The small nucleolar RNA U6 was used as an endogenous control for the normalization of RNA input. MiRNA expression levels were calculated by relative quantification using ABI 7500 Real-Time PCR SDS 1.2 software (Applied Biosystems, Santa Clara, CA, USA), and the fold change of expression $\mid \log _{2}$ (Control / UVB-SIPS) $\mid \geq 2$ were considered as significantly changes. The PCR reaction without template served as a negative control.

\section{Design and subcloning of short hairpin siRNA template into lentiviral vector}

A third generation of self-inactivating lentivirus vector containing a CMV-driven GFP reporter and a H1 promoter upstream of cloning restriction sites (ClaI and mluI) to allow the introduction of oligonucleotides encoding short hairpin RNAs (shRNAs) was provided by Genechem (Shanghai, China). The hairpin consists of a $T$, a 21 nt sense sequence, a short spacer (TTCAAGAGA), an antisense sequence, 6 Ts (a stop signal for RNA polymerase III) and an mluI site. Oligos were annealed and inserted between the mluI and ClaI sites of the plasmid. The shDNA sequence for constructing lentiviruses against human pre-miR-34c-5p (Lenti-siR-miR-34c-5p) was $5^{\prime}$ AGGCAGUGUAGUUAGCUGAUUGC- $3^{\prime}$ and $5^{\prime}$ AAUCACUAACCACACGGCCAGG-3'. Correct insertions of shRNA cassettes were confirmed by restriction mapping and direct DNA sequencing. 


\section{Lentivirus production}

Recombinant lentiviruses were produced by co-transfecting 293T cells with the lentivirus expression plasmid and packaging plasmids using calciumphosphate method. Infectious lentiviruses were harvested at 48 and $72 \mathrm{~h}$ post transfection, centrifuged to eliminate cell debris, and then filtered through $0.22-\mu \mathrm{m}$ cellulose acetate filters. Infectious titer was determined by fluorescence-activated cell sorting analysis of green fluorescent protein (GFP) positive in 293 cells. Virus titers were in the range of $10^{8}$ transducing units $/ \mathrm{mL}$ medium.

\section{Lentiviral vector transductions}

On the day of transduction, NHSFs were replated at $2 \times 10^{4}$ cells/well in 12-well plates along with recombinant lentivirus Lenti-siR-miR-34c-5p at different multiplicities of infection (MOIs) in serum-free growth medium containing $5 \mathrm{mg} / \mathrm{mL}$ polybrene at 37 ${ }^{\circ} \mathrm{C}$ and $5 \% \mathrm{CO}_{2}$. After $4 \mathrm{~h}$, serum containing growth medium was added to the cells, and there was complete replacement of growth medium after $48 \mathrm{~h}$. GFP expression was examined by fluorescent microscopy after 8 days. NHSFs were plated in six-well plates at a density of $1 \times 10^{5}$ cells per well and transfected with Lenti-siR-miR-34c-5p at an MOI of 100 as described above. Empty virus vector control plasmids was employed as vector control and equal volume saline as negative control.

\section{Assay for E2F3 over-expression}

Eukaryote pIRES-E2F3 expression vector was generated by inserting the open reading frame of E2F3a cDNA into the Cla I/EcoR I site of the pIRES1 neo vector (Clontech Laboratories, Inc., Mountain View, CA, USA). Fibroblasts were seeded into 6-well plates at a concentration of $0.5 \times 10^{5}$ /well and transfected with $0.5 \mu \mathrm{g} /$ well of pIRES1 neo as a control vector or pIRES-E2F3 expression vector by using Lipofectamine RNAiMAX. The effects manifested by E2F3 over-expression were assayed at 7 days after the transfection with plasmids.

\section{Cell cycle detection}

Fibroblasts were fixed with $70 \%$ alcohol, washed twice with PBS, digested with RNase, and stained with propidium iodide (PI). A flow cytometer (FAC-Scan, BD, NJ, USA) was used to gather data and images, to analyze the cell cycle, and to calculate the percentage of cells in the G1 phase.

\section{Bioinformatic analysis of miR-34c-5p target genes}

Putative miR-34c-5p targets were predicted using several different algorithms, including TargetScan (http://www.targetscan.org/), Pictar (http:// pictar. bio.nyu.edu/) and miRanda (http://microrna. sanger.ac.uk/). An interaction between miR-34c-5p and the 3' UTR of its target gene was predicted by RNAhybrid (http://bibiserv.techfak.uni-bielefeld. de/rnahybrid/).

\section{Dual-luciferase assay}

The 3' UTR of human E2F3, with one predicted miR-34c-5p binding site mutations (2730-2737, Figure $4)$, was inserted into the multicloning site of the pMir-Luc-target vector plasmid (Shanghai Bioladder Co., Ltd, Shanghai, China). The miR-34c-5p expression clone (miR-34c-5p) was constructed downstream a CMV promoter, and a luciferase assay was performed (Luc-Pair miR Luciferase Assay Kit; GeneCopoeia). The cells were plated in six wells and incubated until $70 \%$ confluent and transfected with one of the following 6 combinations: (1) E2F3 3' UTR (2730-2737); (2) E2F3 3' UTR with miR-34c-5p 2730-2737 seed-matching mutation (E2F3 3' UTR (2730-2737) mt); (3) E2F3 3' UTR (2730-2737) + sc-miR (miRNA scramble control); (4) E2F3 $3^{\prime}$ UTR (2730-2737) mt + sc-miR; (5) E2F3 3' UTR (2730-2737) + miR-34c-5p; (6) E2F3 3' UTR (2730-2737) $\mathrm{mt}+$ miR-34c-5p. The cells were transferred to a 96-well plate 18 hours after transfection and cultured for another $24 \mathrm{~h}$. Both firefly luciferase and Renilla luciferase activities were determined in the NHSFs. Firefly luciferase activity was then normalized with Renilla luciferase activity in the same well.

\section{Detection of E2F3, P2I WAF-I, and P53 expres- sion}

The primer sequence and size of PCR products of target genes are listed in Table 1. U6 primers were used as internal RNA loading and amplification controls. Primary antibodies against E2F3 (Santa Cruz Biotechnology, Santa Cruz, CA), p21 WAF-1 (Cell Signaling Technology, California, USA), P53 (Cell Signaling Technology, California, USA), and $\beta$-actin (Biotime, Haimen, China) were used in western blot analysis.

\section{Statistical analysis}

All analyses were performed with SPSS 13.0 (SPSS Corporation, Chicago, CA, USA). Statistical significance of multiple treatments was determined by analysis of variance (ANOVA) test. $\mathrm{P}<0.05$ was considered to be significant. 
Table I. The primer sequence of target genes.

\begin{tabular}{|c|c|c|}
\hline \multicolumn{2}{|l|}{ Gene name } & \multirow{2}{*}{$\begin{array}{l}\text { primer sequences } \\
\text { 5`- CGGTCATCAGTACCTCTCAGA-3` }\end{array}$} \\
\hline E2F3 & $\begin{array}{l}\text { Forward primer: } \\
\text { Reverse primer: }\end{array}$ & \\
\hline p53 & $\begin{array}{l}\text { Forward primer: } \\
\text { Reverse primer: }\end{array}$ & $\begin{array}{l}\text { 5- CCGCAGTCAGATCCTAGCG -3`} \\
\text { 5- AATCATCCATTGCTTGGGACG -3` }\end{array}$ \\
\hline p21 & $\begin{array}{l}\text { Forward primer: } \\
\text { Reverse primer: }\end{array}$ & 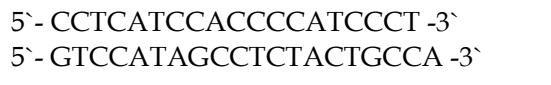 \\
\hline GAPDH & $\begin{array}{l}\text { Forward primer: } \\
\text { Reverse primer: }\end{array}$ & 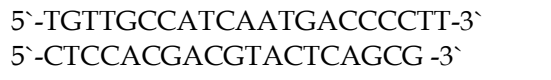 \\
\hline MiR-34c-5p & Forward primer: & 5`-AGGCAGTGTAGTTAGCTGATTGC-3` \\
\hline U6 & $\begin{array}{l}\text { Forward primer: } \\
\text { Reverse primer: }\end{array}$ & $\begin{array}{l}\text { 5`ATTGGAACGATACAGAGAAGATT-3` } \\
\text { 5-GGAACGCTTCACGAATTTG-3` }\end{array}$ \\
\hline
\end{tabular}

Table 2. Differently expressed miRNAs in UVB-induced premature senescence of human dermal fibroblasts

\begin{tabular}{llll}
\hline \multirow{2}{*}{ miRNAs } & \multicolumn{2}{l}{$\log _{2}$ (Control / UVB-SIPS) } \\
\cline { 2 - 3 } hsa-miR-1224-3p & Control & UVB-SIPS & \\
hsa-miR-197-3p & 419.357776 & 1692.797989 & 2.013156 \\
hsa-miR-1976 & 2149.208603 & 10773.654995 & 2.32563 \\
hsa-miR-23a-5p & 1103.044789 & 4707.197797 & 2.093377 \\
hsa-miR-34c-5p & 1033.895368 & 4379.935474 & 2.082819 \\
hsa-miR-4701-5p & 639.074483 & 2801.90345 & 2.132351 \\
hsa-miR-574-5p & 658.034808 & 3367.663795 & 2.355512 \\
hsa-miR-766-3p & 327.902091 & 1370.018711 & 2.062859 \\
hsa-miR-1185-1-3p & 1964.066606 & 8691.728655 & 2.145799 \\
hsa-miR-1185-2-3p & 1599.359178 & 311.123359 & -2.361935 \\
hsa-miR-4638-5p & 1502.326927 & 262.706468 & -2.515675 \\
hsa-miR-4695-5p & 1734.31208 & 375.679215 & -2.20679 \\
hsa-miR-933 & 5210.743432 & 979.097142 & -2.411965 \\
\hline
\end{tabular}

\section{Results and Discussion}

In order to screen the differentially expressed miRNA in UVB-SIPS, we compared the miRNA expression pattern in UVB-SIPS with untreated control in dermal fibroblast. In total, 8 miRNAs were up-regulated and 5 miRNAs were down-regulated after a total of $250 \mathrm{~mJ} / \mathrm{cm}^{2} \mathrm{UVB}$ irradiation at 48 hours after last irradiation (Table 2).

MiR-34c-5p expression was further confirmed to be increased significantly $(\mathrm{P}<0.05)$ after UVB treatment by qRT-PCR detection (Figure 1). Then, we transfected Lenti-siR-miR-34c-5p or Empty Virus Vector into fibroblasts and named them as fibroblasts-siR-miR-34c-5p and fibroblasts-vector, respectively. We used qRT-PCR to confirm the expression level of miR-34c-5p (Figure 2).

To explore the role of miR-34c-5p in cellular senescence induced by UVB, fibroblasts-vector and fibroblasts-siR-miR-34c-5p were treated with repeated UVB treatments. As expected, after treatment, fibro- blasts-vector irradiated cells became enlarged and irregular in shape, and the percentage of SA- $\beta$-gal positive cells increased (Figure $3 b ; \mathrm{P}<0.05$. fibroblasts-vector: $\quad 8.03 \pm 1.32 \%$, fibroblasts-vector+UVB-SIPS: $90.83 \pm 2.92 \%)$. However, underexpression of miR-34c-5 $p$ alleviated the morphological changes induced by repeated UVB treatments (Figure 3a). There were less SA- $\beta$-gal positive cells in fibroblasts-siR-miR-34c-5 $p$ than that in fibroblasts-vector after UVB treatment (Figure 3b; $\mathrm{P}<0.05$. fibroblasts-siR-miR-34c-5p+UVB-SIPS: $36.88 \pm 5.53 \%$ ). Our data provided evidence that the down-regulation of miR-34c-5p in human dermal fibroblasts can delay UVB-induced premature senescence. In good agreement with our report, Kumamoto et al. showed that after the administration of the MDM2 inhibitor, Nutlin-3, to human diploid fibroblasts, induction of miR-34c as well as senescence was observed(10).

To assess whether the over-expression of miR-34c-5p affects the G1 arrest in fibroblasts after UVB-SIPS, the percentage of cells in G1 phase were 
measured. The level of G1 phase was increased in fibroblasts-vector+UVB-SIPS. However, down expression of miR-34c-5p antagonized the above change (Figure 3c).

Compared with classical transcriptional factors, miRNAs act mainly via regulation of their target genes at the mRNA level. For example, regulation of microtubule-associated protein tau by miR-34c-5p determines the chemosensitivity of gastric cancer to paclitaxel (11). miR-34c-5p directly targets sGCbeta under hypoxia (12). Gluconeogenesis is severely compromised in hepatocellular carcinoma (HCC) by IL6-Stat3-mediated activation of miR-23a, which directly targets PGC-1a and G6PC, leading to decreased glucose production (13). With the help of current bioinformatics software, we proposed that E2F3, which play an essential role in cell cycle progression, proliferation, and development, is the target of miR-34c-5p. Moreover, dual-luciferase assay analysis indicated that the predicted $2730-2737$ binding site was functional in vitro (Figure 4). To further confirm the effect of miR-34c-5p on E2F3 expression, we detected the mRNA and protein levels of E2F3 under miR-34c-5p down-expression condition after UVB-SIPS. We found that the expression of E2F3 decreased significantly in UVB-SIPS and then they were rescued by miR-34c-5p down-expression in fibroblasts-siR-miR-34c-5p after repeated UVB treatments (Figure 5; $\mathrm{P}<0.05$ ). These results indicated that $\mathrm{E} 2 \mathrm{~F} 3$ is a real down-stream target of miR-34c-5p.

It is reported that activation of the p53 pathway could be caused as a consequence of the repression of the E2F3 as in the case of fibroblast with knockout of E2F3 (14-16). It is well-established that the p53 pathway is involved in aging and photoaging (17). Active p53 is known to trigger p21 WAF-1 overexpression (18). P21WAF-1 is a cyclin dependent kinase inhibitor that blocks the cell cycle in G1 phase (19), which corresponds to the cell cycle analysis results. Supporting these assumptions, we confirmed that UVB-SIPS increased the expression of p21 ${ }^{\mathrm{WAF}-1}$ and $\mathrm{P} 53$ expression in cultured fibroblasts. To further investigate the mechanism of miR-34c-5p in UVB-induced senescence, we explored the effects of miR-34c-5p on p21 ${ }^{\text {WAF-1 }}$ and P53 expression in UVB-SIPS fibroblasts. We found that the expression of p21 ${ }^{\mathrm{WAF}-1}$ and P53 were decreased by miR-34c-5p under-expression in fibroblasts-siR-miR-34c-5p after repeated UVB treatments (Figure 5; $\mathrm{P}<0.05$ ). Moreover, over-expressed E2F3, which is confirmed to be the direct target of
miR-34c-5p, can rescue fibroblasts from UVB induced premature senescence. As indicated by our results, there were less SA- $\beta$-gal positive cells in pIRES-E2F3 than that in pIRES1-vector after UVB treatment (Figure 6c; $\mathrm{P}<0.05$, UVB-SIPS+pIRES-E2F3: $54.82 \pm$ $4.55 \%)$. In light of our results, it seems reasonable to conclude that decreased levels of miR-34c-5p may contribute to up-regulated E2F3 which sequentially lead to inactivation of p53-p21 pathway thus interfering with UVB-SIPS induced senescence. It has been shown that active p53 directly induces miR-34c-5p expression $(20,21)$. In this consideration, miR-34c-5p in turn indirectly increases p53 activity, thus establishing a positive feedback loop leading to cell-cycle arrest. However, additional experiments will be required to confirm this interpretation.

The major damage of photoaged skin is related to the connective tissue of the dermal compartment with quantitative and qualitative alterations of the dermal extracellular matrix (22). It has been reported that following chronic ultraviolet stress treatment, the expression of interstitial collagenase/matrix metalloproteinase- 1 is induced. This induction is prolonged in the period of growth arrest phase in dermal fibroblasts, while tissue inhibitor of metalloproteinase-1, the major inhibitor of matrix-metalloproteinase-1, is only slightly induced (23). This imbalance between matrix-degrading metalloproteinases and their inhibitors may lead to connective tissue damage, a hallmark of premature aging. These findings, together with our observation that miR-34c-5p plays an important role in cellular senescence, suggesting that miR-34c-5p may also be involved in photoaging of the skin.

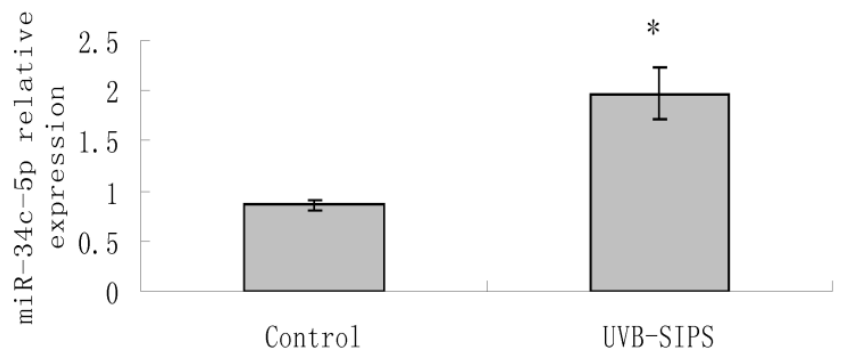

Figure I. qRT-PCR confirmation of miR-34c-5p expression in UVB-induced premature senescence of human dermal fibroblasts. Results are shown as means $\pm S D(n=3)$. $* P<0.05$ compared with control group. Bars, mean \pm standard error. 


\section{A}

light

Fibrobalsts -vector

fluorescence
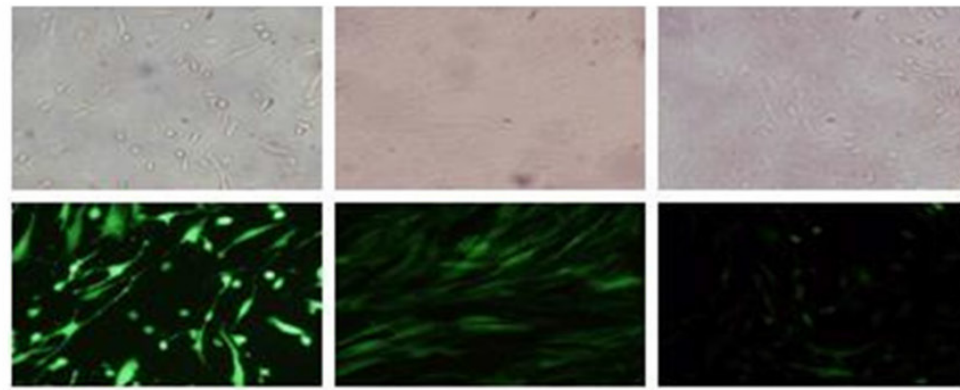

$\mathrm{MOI}=\mathbf{1 0 0}$

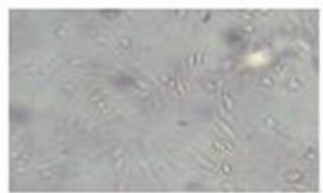

$\mathrm{MOI}=\mathbf{1 0}$

MOI=1

light

Fibrobalsts

-siR-miR-34c-5P
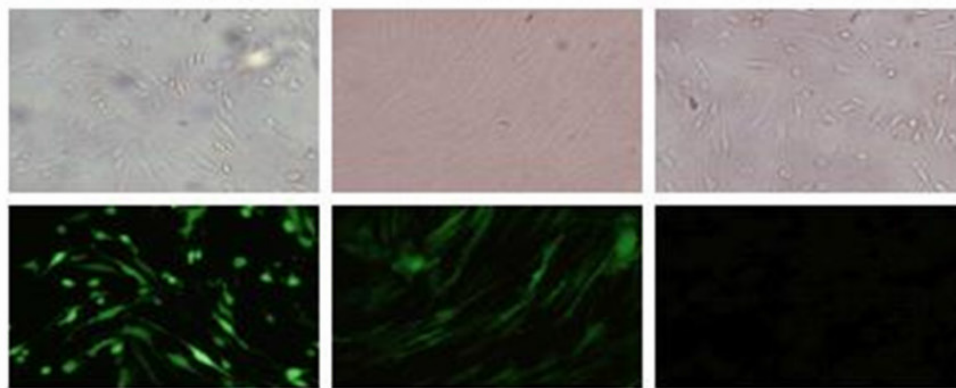

fluorescence
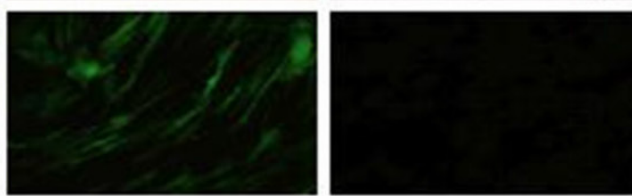

B

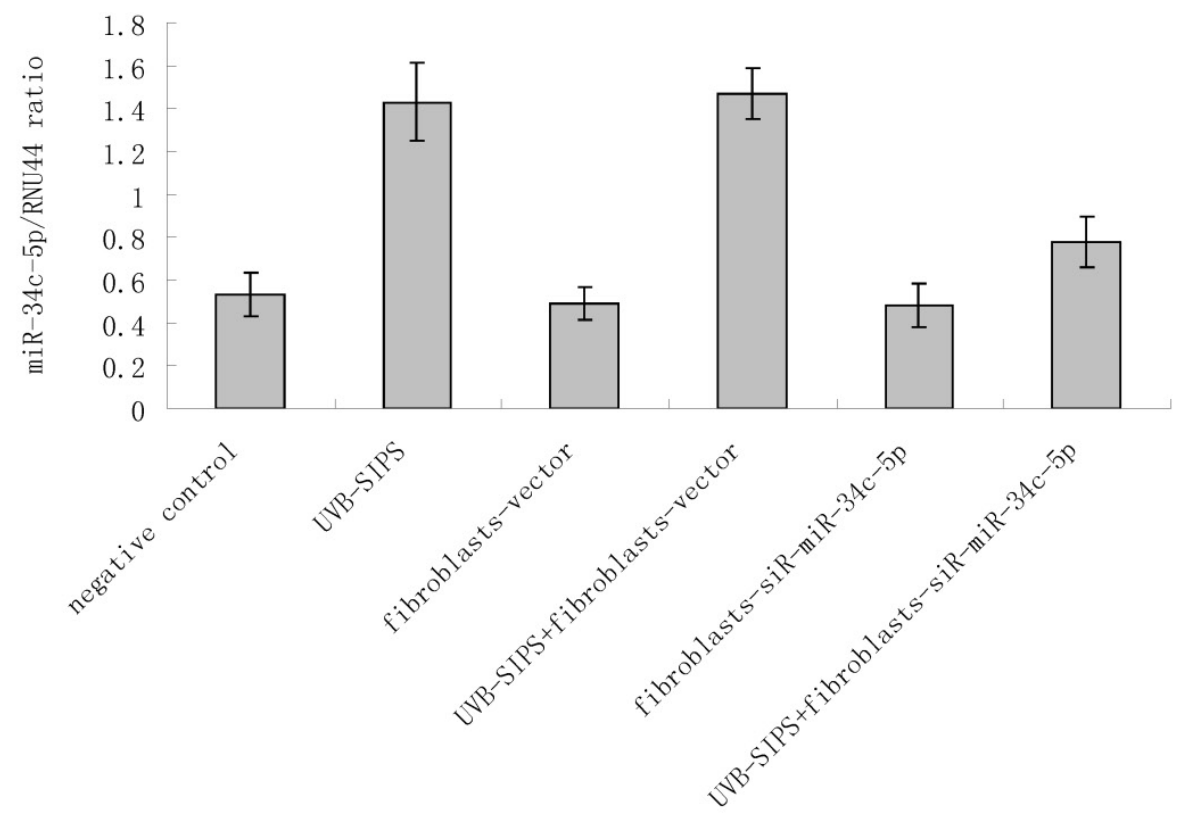

Figure 2. Determination of lentiviral transduction efficiency and lentivirus-mediated miR-34c-5p down-expression in fibroblasts. (A) Transduction efficiency was estimated 8 days after infecting at indicated MOIs. GFP expression was observed under light microscopy (up), fluorescence microscopy (down). The highest GFP expression of NHSFs appeared with MOI of 100. (B) MiR-34c-5p was underexpressed by lentivirus transfection in dermal fibroblasts and confirmed by qRT-PCR. Results are shown as means \pm SD $(n=3)$. 


\section{A Negative control}

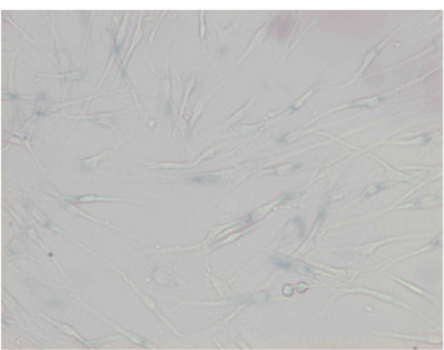

UVB-SIPS+ fibroblasts-vector

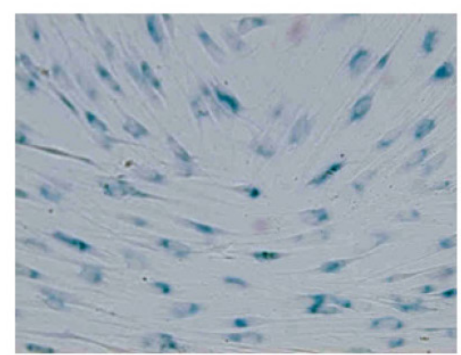

UVB-SIPS

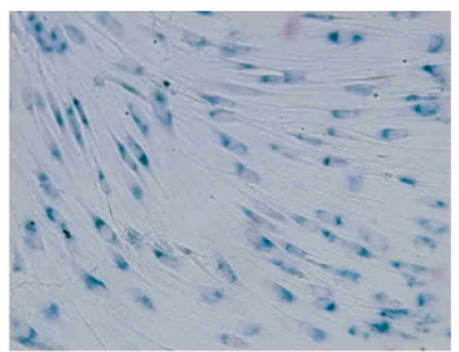

Fibroblasts-siR-miR-34c-5p
Fibroblasts-vector

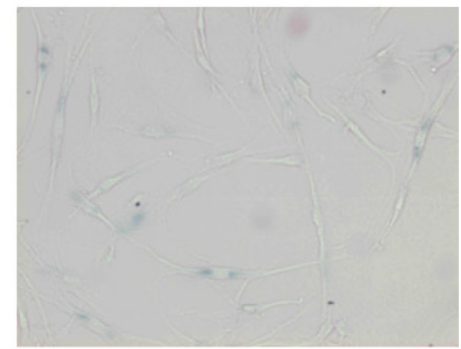

UVB-SIPS+

fibroblasts-siR-miR-34c-5p
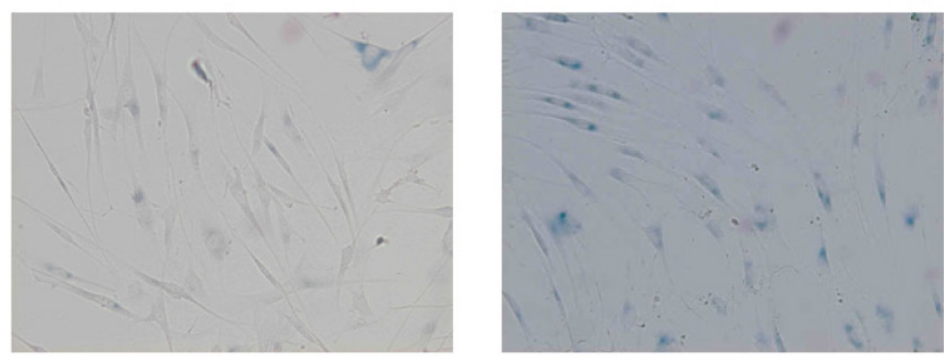

B

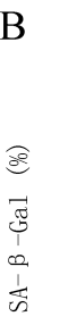

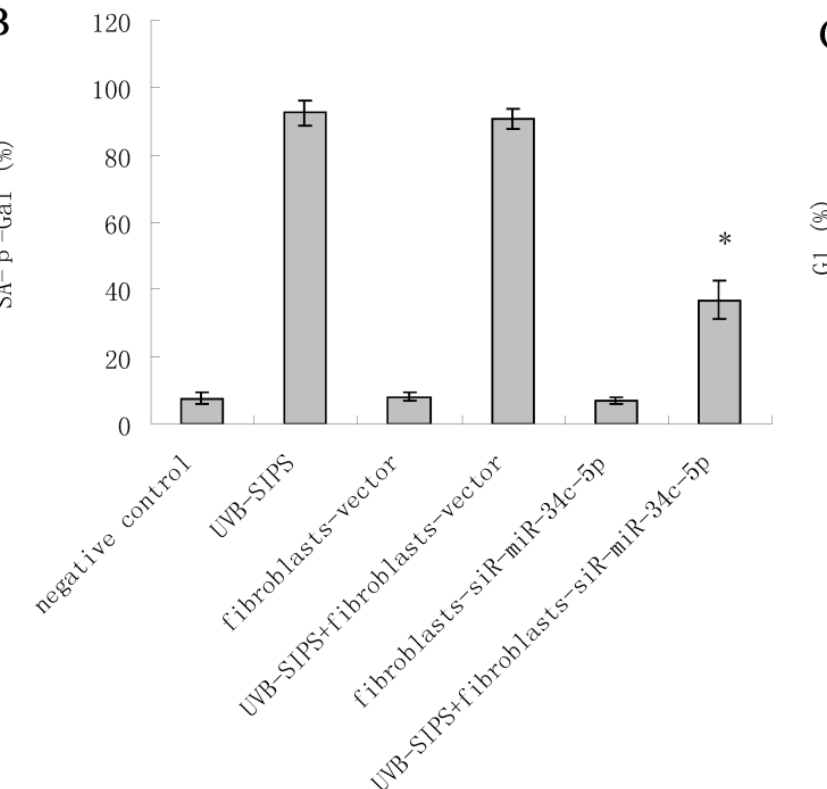

C 90

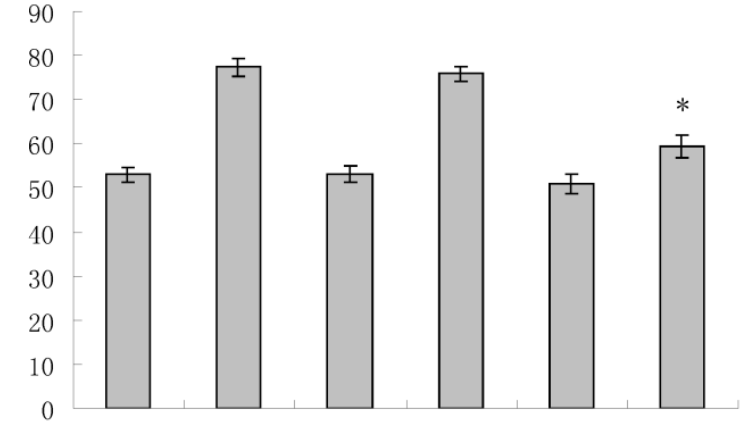

Figure 3. MiRNA underexpression delayed premature senescence induced by repeated subcytotoxic UVB treatments in normal human dermal fibroblasts. (a) The SA- $\beta$-gal staining in fibroblasts-vector and fibroblasts-siR-miR-34c-5p after repeated UVB treatments. (b) The SA- $\beta$-gal positive cells were increased significantly in non-transfected and fibroblasts-vector after repeated UVB treatments, and there were less SA- $\beta$-gal positive cells in fibroblasts-siR-miR-34c-5p than that in fibroblasts-vector after repeated UVB treatments. Results are shown as means $\pm S D(n=3)$. (c) The GI phase cells were increased significantly in non-transfected and fibroblasts-vector after repeated UVB treatments, and there were less GI phase cells in fibroblasts-siR-miR-34c-5p than that in fibroblasts-vector after repeated UVB treatments. Results are shown as means $\pm S D(n=3)$. *P $<0.05$, compared with UVB-SIPS and UVB-SIPS+fibroblasts-vector. 

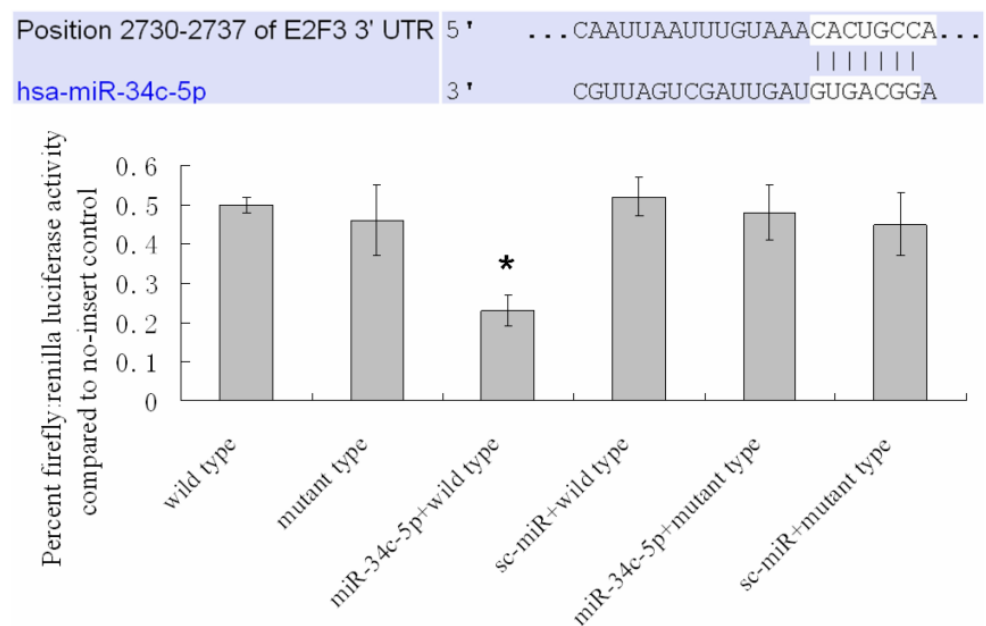

Figure 4. MiR-34c-5p targets the E2F3 3' UTR. Mutation of a predicted miR-34c-5p binding site in the E2F3 3' UTR (2730-2737) abrogated the repressive effect of miR-34c-5p on the luciferase activity of a E2F3 3' UTR-luciferase reporter. Results are shown as means $\pm S D(n=3)$. *P $<0.05$ versus wild type.
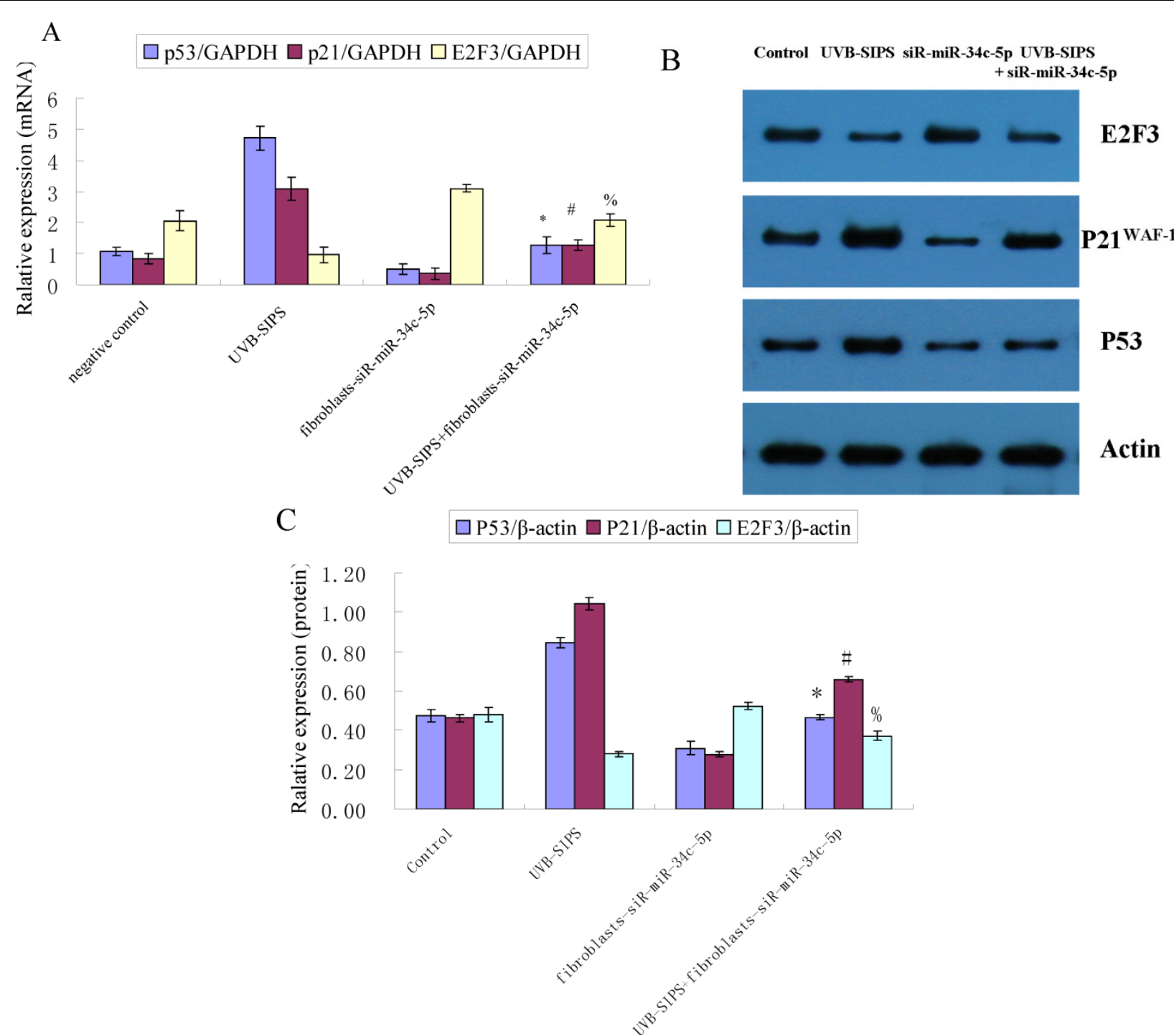

Figure 5. Effect of miR-34c-5p downexpression on E2F3, p2 $\left.\right|^{\text {WAF-1 }}$ and P53 expression in UVB-SIPS fibroblasts. (a) $q R T-P C R$ analysis of E2F3, p2 ${ }^{\text {WAF-1 }}$ and P53 in control, UVB-SIPS, fibroblasts-siR-miR-34c-5p and UVB-SIPS+ fibroblasts-siR-miR-34c-5p. Results are shown as means \pm SD ( $n=$ 3). (b) Western blotting analysis of E2F3, P2 $I^{\text {WAF-I }}$ and P53 level in control, UVB-SIPS, fibroblasts-siR-miR-34c-5p and UVB-SIPS+ fibroblasts-siR-miR-34c-5p. (c) Blots from triplicate experiments were scanned, and densitometric values corrected for actin were plotted as mean \pm SD. $* P<$ $0.05, " P<<0.05, \% P<0.05$ compared with UVB-SIPS. 

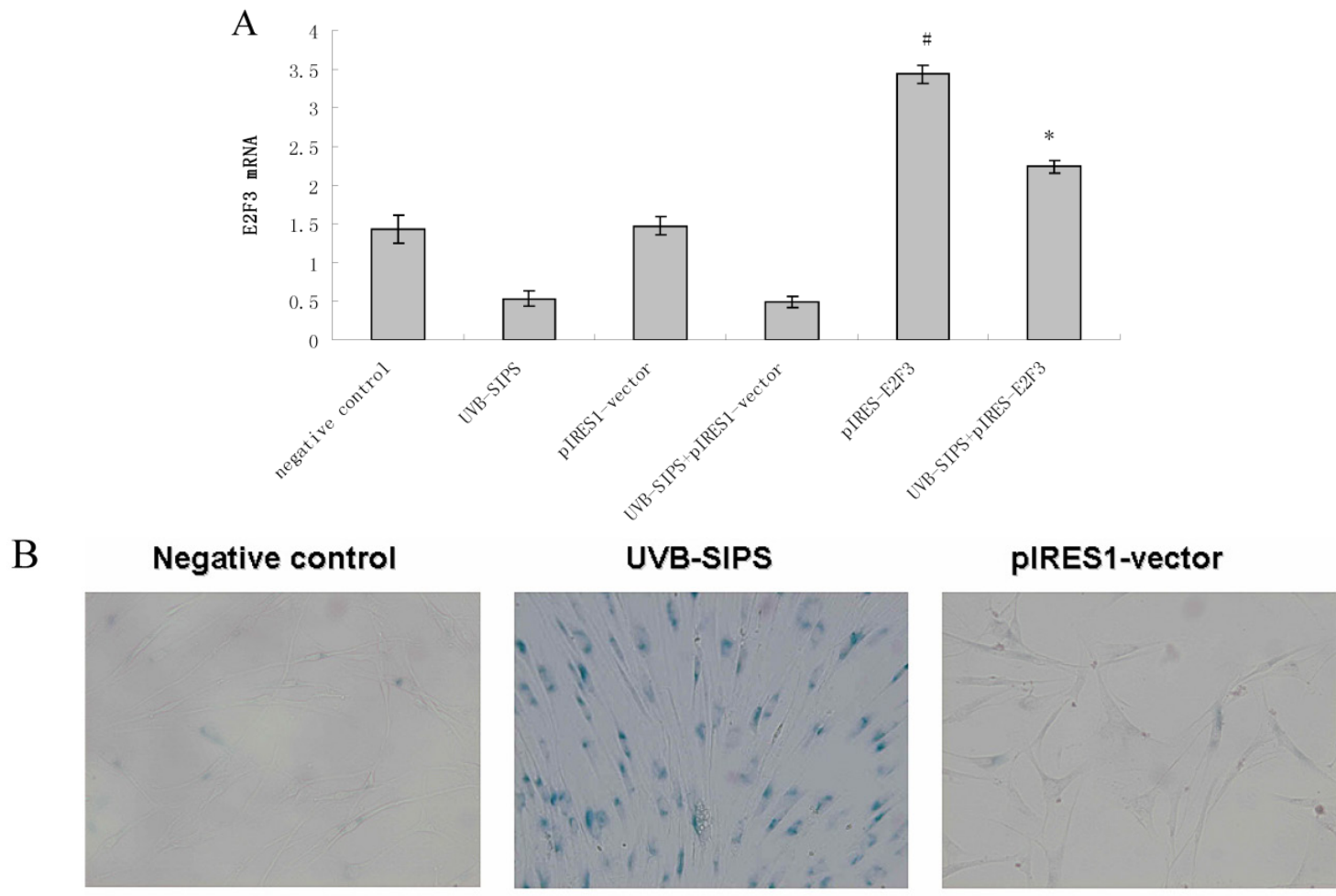

\section{UVB-SIPS+ pIRES1-vector}

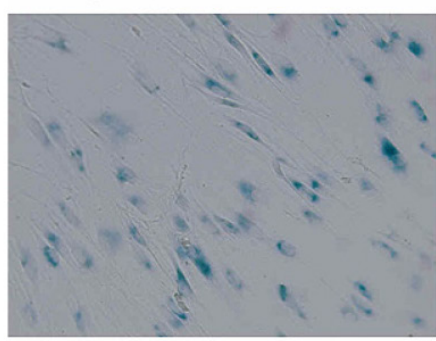

C 100

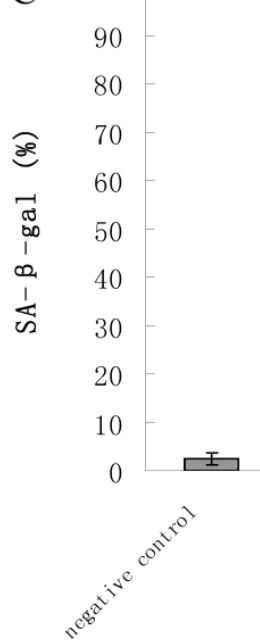

pIRES-E2F3
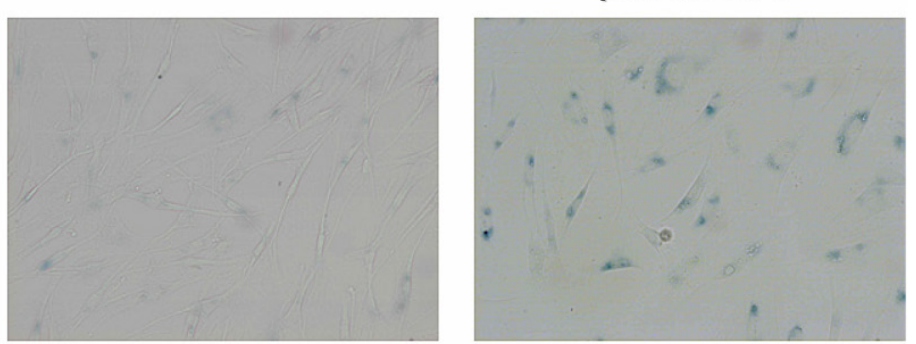

UVB-SIPS+

pIRES-E2F3

Figure 6. Enforced expression of E2F3 delayed premature senescence induced by repeated subcytotoxic UVB treatments in normal human dermal fibroblasts. (a) E2F3 was over-expressed by pIRES-E2F3 vector transfection in dermal fibroblasts after repeated UVB treatments and confirmed by qRT-PCR. (b) The SA- $\beta$-gal staining in pIRES-E2F3 or pIRESI-vector transfected fibroblasts after repeated UVB treatments. (c) The SA- $\beta$-gal positive cells were increased significantly in non-transfected and pIRESI-vector after repeated UVB treatments, and there were less SA- $\beta$-gal positive cells in pIRES-E2F3 transfected fibroblasts than that of in pIRESI-vector group after repeated UVB treatments. Results are shown as means $\pm S D$ ( $n=3$ ). ${ }^{\#}<<0.05$, compared with PIRESI-vector and negative control. *P < 0.05, compared with UVB-SIPS and UVB-SIPS+pIRESI-vector. 


\section{Conclusions}

We found that several miRNAs, including miR-34c-5p, are dysregulated in UVB-SIPS, and down-regulation of miR-34c-5p can delay senescence of human dermal fibroblasts induced by UVB treatment. Further study elucidate that E2F3 is the downstream target of miR-34c-5p. E2F3 regulated P53-P21 pathway play a role in regulating the procedure of UVB-SIPS. We believe that the relationships of miR-34c-5p and some senescence-related proteins, and the exact mechanisms of miR-34c-5p in senescence might be a novel and potent way to study cellular senescence and photoaging.

\section{Acknowledgments}

This work was supported by grant from the China National Natural Science Foundation (81000700 and 81171518), science project from traditional Chinese medicine Bureau of Jiangsu Province (LZ11084), and Jiangsu National Natural Science Foundation (BK2012877 and BK2012168).

\section{Competing Interests}

The authors have declared that no competing interest exists.

\section{References}

1. Debacq-Chainiaux F, Borlon C, De Hertogh B, Remacle J, Morvan PY, Vallee R, Toussaint O. Identification of potential anti-photoageing algal compounds using an in-vitro model of photoageing. J Pharm Pharmacol. 2006;58(12):1577-83.

2. Chainiaux F, Magalhaes JP, Eliaers F, Remacle J, Toussaint O. UVB-induced premature senescence of human diploid skin fibroblasts. Int J Biochem Cell Biol. 2002; 34(11):1331-9.

3. Zhou BR, Xu Y, Permatasari F, Liu WL, Li W, Guo XF, Huang QH, Guo $Z$, Luo D. Characterization of the miRNA profile in UVB-irradiated normal human keratinocytes. Exp Dermatol. 2012;21(4):317-9.

4. Li G, Luna C, Qiu J, Epstein DL, Gonzalez P. Alterations in microRNA expression in stress-induced cellular senescence. Mech Ageing Dev. 2009; 130(11-12):731-41.

5. He L, He X, Lim LP, de Stanchina E, Xuan Z, Liang Y, Xue W, Zender L, Magnus J, Ridzon D, Jackson AL, Linsley PS, Chen C, Lowe SW, Cleary MA, Hannon GJ. A microRNA component of the p53 tumour suppressor network. Nature. 2007; 447(7148):1130-4.

6. Lafferty-Whyte K, Cairney CJ, Jamieson NB, Oien KA, Keith WN. Pathway analysis of senescence-associated miRNA targets reveals common processes to different senescence induction mechanisms. Biochim Biophys Acta. 2009;1792(4):341-52.

7. Zhou BR, Xu Y, Wu D, Permatasari F, Gao YY, Luo D. Ginsenoside Rg1 protects human fibroblasts against psoralen- and UVA-induced premature senescence through a telomeric mechanism. Arch Dermatol Res. 2012; 304(3):223-8.

8. Oh S, Lee E, Lee J, Lim Y, Kim J, Woo S. Comparison of the effects of $40 \%$ oxygen and two atmospheric absolute air pressure conditions on stress-induced premature senescence of normal human diploid fibroblasts. Cell Stress Chaperones. 2008;13(4):447-58.

9. Liu CG, Calin GA, Meloon B, Gamliel N, Sevignani C, Ferracin M, Dumitru CD, Shimizu M, Zupo S, Dono M, Alder H, Bullrich F, Negrini $\mathrm{M}$, Croce CM. An oligonucleotide microchip for genome-wide microRNA profiling in human and mouse tissues. Proc Natl Acad Sci USA 2004;101(26):9740-4.

10. Kumamoto K, Spillare EA, Fujita K, Horikawa I, Yamashita T, Appella E, Nagashima M, Takenoshita S, Yokota J, Harris CC. Nutlin-3a activates p53 to both down-regulate inhibitor of growth 2 and up-regulate mir-34a, mir-34b, and mir-34c expression, and induce senescence. Cancer Res. 2008;68(9):3193-203.

11. Wu H, Huang M, Lu M, Zhu W, Shu Y, Cao P, Liu P. Regulation of microtubule-associated protein tau (MAPT) by miR-34c-5p determines the chemosensitivity of gastric cancer to paclitaxel. Cancer Chemother Pharmacol. 2013 May;71(5):1159-71.

12. Xu X, Wang S, Liu J, Dou D, Liu L, Chen Z, Ye L, Liu H, He Q, Raj JU, Gao Y. Hypoxia induces downregulation of soluble guanylyl cyclase beta1 by miR-34c-5p. J Cell Sci. 2012;125(Pt 24):6117-26.

13. Wang B, Hsu SH, Frankel W, Ghoshal K, Jacob ST. Stat3-mediated activation of microRNA-23a suppresses gluconeogenesis in hepatocellular carcinoma by down-regulating glucose-6-phosphatase and peroxisome proliferator-activated receptor gamma, coactivator 1 alpha. Hepatology. 2012; 56(1):186-97.

14. Sharma N, Timmers C, Trikha P, Saavedra HI, Obery A, Leone G. Control of the p53-p21CIP1 Axis by E2f1, E2f2, and E2f3 is essential for G1/S progression and cellular transformation. J Biol Chem. 2006; 281(47):36124-31.

15. Wu L, Timmers C, Maiti B, Saavedra HI, Sang L, Chong GT, Nuckolls F, Giangrande P, Wright FA, Field SJ, Greenberg ME, Orkin S, Nevins JR, Robinson ML, Leone G. The E2F1-3 transcription factors are essential for cellular proliferation. Nature. 2001;414(6862):457-62.

16. Timmers C, Sharma N, Opavsky R, Maiti B, Wu L, Wu J, Orringer D, Trikha P, Saavedra HI, Leone G. E2f1, E2f2, and E2f3 control E2F target expression and cellular proliferation via a p53-dependent negative feedback loop. Mol Cell Biol. 2007; 27(1):65-78.

17. Kosmadaki MG, Gilchrest BA. The role of telomeres in skin aging/photoaging. Micron. 2004; 35(3):155-9.

18. Li GZ, Eller MS, Firoozabadi R, Gilchrest BA. Evidence that exposure of the telomere 3 ' overhang sequence induces senescence. Proc Natl Acad Sci USA. 2003; 100(2):527-31.

19. Sherr CJ, Roberts JM. CDK inhibitors: positive and negative regulators of G1-phase progression. Genes Dev. 1999; 13(12):1501-12.

20. Corney DC, Hwang CI, Matoso A, Vogt M, Flesken-Nikitin A, Godwin AK, Kamat AA, Sood AK, Ellenson LH, Hermeking H, Nikitin AY. Frequent downregulation of miR-34 family in human ovarian cancers. Clin Cancer Res. 2010;16(4):1119-28.

21. Hermeking H. The miR-34 family in cancer and apoptosis. Cell Death Differ. 2010;17(2):193-9.

22. Yaar M, Gilchrest BA. Skin aging: postulated mechanisms and consequent changes in structure and function. Clin Geriatr Med. 2001; 17(4):617-30.

23. Wlaschek M, Ma W, Jansen-Durr $P$, Scharffetter-Kochanek $K$. Photoaging as a consequence of natural and therapeutic ultraviolet irradiation--studies on PUVA-induced senescence-like growth arrest of human dermal fibroblasts. Exp Gerontol. 2003; 38(11-12):1265-70. 